\title{
Teaching medical students a difficult subject - child abuse
}

\author{
Eileen Vizard, Consultant Child Psychiatrist, Newham Child Guidance Clinic and \\ Lecturer, The London Hospital, London E1.
}

As a specialist in child abuse for the past five years, I have been presented with various technical diffculties in trying to convey important, but often emotive, material to medical students and, for that matter, to groups of professional colleagues from other disciplines. It is hoped that the following reflections may be useful to colleagues who teach medical students about child abuse.

\section{Advantages in teaching a difficult subject}

Are there any advantages in teaching a difficult subject, as opposed to something rather less worrying or contentious? In relation to child abuse, I think the answer is yes.

\section{Pros}

Intellectual challenge Links with the Law

Reparative wish

Child-centred urgency

\begin{tabular}{l}
\multicolumn{1}{c}{ Cons } \\
Strong emotions aroused \\
Emotional blocking \\
Attitudes \\
Apostolic \\
Cynical withdrawal \\
Intellectualisation \\
Rescue fantasies
\end{tabular}

Firstly, there is the intellectual challenge to the medical student of coming to terms with a multifaceted and chronic problem. This subject presents fascinating medical diagnostic issues, challenging legal evidential points and, importantly, major ethical issues around professional intervention in such cases. Intellectually, therefore, this difficult subject has the advantage of not being dull.

Secondly, because of the nature of child abuse, a strong reparative wish, i.e. a wish to understand in order to make things better, is often awoken in the medical student while learning. This can be seen as a reasonable motivation for learning, as long as it is kept under control and does not tip over into 'rescue fantasies' which are pointless. Another advantage in teaching this difficult subject is that it involves children and therefore, in the mind of the medical student, there may rightly be a sense or urgency to understand in order to get something done quickly.

As with all teaching, the subject matter needs to be properly integrated into the curriculum in a way which allows for reinforcement and 'top ups' so that learning will persist. This may be particularly rel- evant for a difficult subject, despite the advantages mentioned earlier. In general terms, I have found the following model for teaching child abuse to be acceptable to medical students, adaptable to short teaching slots as well as intra-curricular courses on the subject.

\section{Verbal presentation}

With verbal presentation, I am assuming that an adequate 'handout' on the subject should be given to each medical student on arrival so that the verbal presentation is not top heavy with statistics. I have found that two or three relevant statistics and one 'buzz' word or concept in relation to child abuse (for example, "frozen watchfulness") will often serve as a perfectly adequate core for talking around the subject. Clinical examples will then allow the subject of child abuse to be brought to life around the chosen core concept.

To avoid overload and switch-off, the bulk of presenting signs and symptoms may need to be presented separately in the handout and brought up in later discussion. Again it does not seem helpful to overload medical students with descriptions of four or five explanatory models for a difficult subject when they may already be struggling with a whole range of very strong feelings about whether or not they wish to listen in the first place at all. Concentration will be better maintained when one explanatory model is described in some detail and others are mentioned in passing and also in the handout.

Medical students will often feel overwhelmed by the nature of the subject shortly after the teaching session starts. I have found it useful to move directly on to describing the sequelae of child abuse as a rationale for helping children in the first place. In other words, by naming the serious sequelae, which many students may feel themselves helpless to prevent in any way, there may be a paradoxical lessening of anxiety in the group, particularly if this is linked to suggestions about treatment and prevention. However, do not make the mistake of talking interminably about these very serious sequelae without linking them to more hopeful possibilities.

Finally, in making a verbal presentation, it is important to remember that this is a serious subject but that one should not fall into the trap of 'student 
abuse' by off-loading all one's own feelings of hopelessness and anger onto a vulnerable captive audience. It is always possible to introduce a lighter moment and the occasional joke in order to preserve the sanity of both teacher and student. In this respect the invention of anatomically correct dolls has proved a wonderful icebreaker in teaching!

\section{Experiental work}

Recent research ${ }^{1}$ has demonstrated the importance of involving medical students in practical active exercises such as the role play of interviews, with video feedback, debating difficult emotional issues, group sculpting, etc. While later work ${ }^{2}$ has rightly sounded a note of caution about the need to put all experiental medical student teaching into an overall, long-term context, nevertheless my own experience is that medical students both value and learn from experiental exercises a great deal more readily than they do from straightforward verbal information.

As a warm-up exercise, it is usually easy for medical students to engage in a sculpt of a child-abusing family set up around various themes of, for example, the consequences of a disclosure of child abuse, or who listens to whom in child-abusing families, or how the abused child can leave home, etc. This will get the students out of their chairs and moving, and leads easily into, for instance, a role-play of the abusing family itself. (It may seem an obvious thing to say but, in my experience, it is not the time for democratic discussion when allocating roles for a role play. Nobody really wants to do role play, and offering a group of anxious ambivalent group of students a choice of roles or no roles at all simply heightens anxiety. It is far better for the teacher to allocate roles firmly, pointing out that there may be an opportunity to 'swap' roles later on in order to experience a different aspect of the family dynamics.)

Similarly, if time allows, an exercise which I have found particularly helpful, and which can 'use up' quite large numbers of medical students, is to role play a child abuse case conference, with the medical students cast into scripted roles of the various multidisciplinary professionals. In order to pinpoint common professional attitudes towards working together, the scripts can be made somewhat stereotyped, i.e. the woolly-minded social worker, the pompous hospital doctor, long-winded psychiatrist etc. A mere hint at the stereotype in the script is usually enough to put students well and truly in role, and is particularly helpful for nervous students by providing a role framework.

A case vignette is given to the group, roles are allocated, and if there are students left over they act as observers of designated aspects of the case conference group process. This exercise is particularly useful in emphasising the future professional role of the medical student and gives an understanding of how to set up, and participate in these important meetings, which will be part of daily work for many newly qualified house officers, whether in casualty out-patient departments, or on the wards. A strong sense of the interprofessional rivalries and communication problems, which have contributed to various child abuse enquiries in the past, can really be brought to life for the medical students during this exercise.

Along the same lines, and perhaps as an exercise for use in a second or third teaching slot with medical students, small groups of students can be asked to devise elementary guidelines for dealing with suspicions or disclosures of child abuse. (Since the various professional bodies concerned with guidelines on response to child abuse have been struggling with this issue for about 20 years, it is only fair to predict failure for the students in relation to this task, and to explain it to them, as an exercise in developing 'means/ends' thinking skills, which it is hoped will stand them in good stead later.)

It is surprising that, despite the prevalence of video as a teaching aid nowadays, there are few studies indicating exactly if and how video is useful in facilitating learning, with the exception of earlier American experiences. ${ }^{3}$ However, the Manchester experience ${ }^{4}$ has indicated that psychiatric trainees, as opposed to medical students, seem to benefit from teaching via videotape in relation to basic psychotherapy skills. More specifically, although the videotaping and subsequent feedback of role play with medical students as described above is useful, this does require a full morning or afternoon session in order to set up equipment etc. and allow time for subsequent discussion.

Having said this, the medical students whom I have taught using this approach thoroughly enjoyed the experience; they seemed to have learned particularly intensively from the use of video feedback of their role play, coupled with the 'freeze frame' technique whereby a frozen moment in the role play is viewed and discussed by the group as a whole. The notion of the frozen moment of a metaphor for family functioning has been described elsewhere, ${ }^{5}$ and if time does not allow extensive video role play by medical students I can recommend the simple technique of viewing a child abusing family assessment/therapy session, with the medical students, pressing the freeze frame button at various points and promoting discussion around questions such as "What do you think the mother will say next?" or "What question should the interviewer now be thinking of asking?" Whilst the medical students' responses may contain elements of projection, I think it is more important that the teacher deals with their responses in a way which emphasises the medical students' professional skills in observing process and being objective. 
Minimum high impact material

Teaching materials may, for convenience, be divided into those with low impact, such as written handouts, books, statistics slides, etc. (i.e. low on impact but high on soporific ratings!) and those with high impact, such as picture slides, audiotapes, and videotapes. Having made this divide, it goes without saying that a good teacher can bring the dullest material to life and a bad teacher, conversely, can send the liveliest student to sleep. Nevertheless, in relation to teaching a difficult subject such as child abuse, a word of caution may be advisable in relation to highimpact teaching materials, such as picture slides and videotapes. The advantages of these high-impact materials are that they show a 'picture' of the childas-a-whole, that certain aspects of the picture may be looked at in detail, that there is immediate feedback from visual or sound material, and that, it is hoped, learning is intensified through the immediacy of this process. However, the disadvantage of this high impact material must not be overlooked.

Continuous showing of explicit disclosure interviews with distressed sexually abused children, or large numbers of colour slides depicting nonaccidental injuries, or post mortem findings, may seem to some observers to have a lurid quality. If a great deal of this material is used in teaching, the student may experience a sense of flooding, of being given too much, and this in turn may lead to the phenomenon of gaze aversion (noted in the supervisor of a child abuse case who wishes to avoid hearing the subject matter presented by a supervisee and who literally averts his or her gaze). This is a reaction which I have noted on numerous occasions now while using high impact videotape or slide material in teaching students. Gaze aversion may lead to attempts to fall asleep in order to avoid seeing and hearing the subject matter in ways which may seem rather bizarre, such as sitting with eyes tightly closed a few feet away from a loudly playing TV monitor. This physical and emotional aversion to the subject may unfortunately lead the student into more denial of the subject's importance and is probably based on an experience of feeling too much in a confused way about the material, and not being able to think or to learn about what is going on. Ultimately, over-use of high impact material may lead to a sense of being deskilled amongst the medical students, based on feelings of hopelessness engendered by the 'over the top' quality of the presentation. Therefore a minimum amount of high impact material followed by an appreciable pause, so that this material can be taken on board and digested, can then lead to maximum discussion/feedback.

\section{A period of maximum discussion/feedback}

In itself, protracted discussion may not necessarily facilitate learning. However, if about 25 minutes or half an hour can be left after the two hour teaching slot, which includes some of the above suggestions, then this should be adequate time to take up unresolved issues with the students. Perhaps teaching of child abuse is rather different from the teaching of, for instance, psychotherapeutic skills, in that cognisance must be taken of the medical student's feelings about the subject, but at the same time this really cannot be the sole focus of the teaching slot. Factual information about how to diagnose the problem, coupled with skills training in how to deal with it, need to go hand in glove with reference to the difficult feelings which this subject will rouse.

\section{Disadvantages of teaching a difficult subject}

What are the disadvantages of teaching such a subject? These may be more apparent than the advantages, and include the very strong emotions aroused by the subject itself. Since child abuse is an umbrella term including physical child abuse, sexual child abuse and emotional abuse, a range of strong emotions may be evoked, such as disgust, excitement, blaming, etc. While these feelings may be present in all of us to greater or lesser extent, their unrecognised presence in medical students attempting to learn about the subject may lead to a conflict about the feelings mentioned. If nothing is done about this conflict, the student may start to deny the importance of the subject itself. In other words, if the feelings are too strong, conflict and switch-off may occur. Finally, a disadvantage facing the teacher may be pre-existing attitudes within the students brought to the session in relation to the subject. These attitudes can include the extremely irritating 'apostolic fervour', belonging, in my experience, to radical feminists of either sex, manifested by an urge to convert everyone to the 'cause' of diagnosing child abuse. Conversely, lawyers manque among the students may wish endless debate about controversial evidential issues, such as false allegations of sexual abuse. Apostolic fervour is often seen in direct contrast to overt cynicism from some medical students about the whole difficult subject and, unlike their senior colleagues, the conflict within these cynical students may be fairly tricky to engage with in a brief teaching slot. It may be that a process of intense identification with the subject matter occurs very readily and if unrecognised by the student may possibly impede learning.

We need to remember that medical students are at the beginning of their training, and that an overly negative approach to the subject may only succeed in pushing them still further into denial and repression. It is probably a good idea to finish every teaching slot 
on child abuse with a check that the students have left with their existing skills reinforced, with a sense of hopefulness that they can develop new skills and attitudes, and with a sense of tolerance towards their old attitudes and those of other people which stem out of reactions to this difficult subject.

\section{References}

${ }^{1}$ Aveline, M. \& Price, J. (1986) The Nottingham experiental day in psychotherapy: A new approach to teaching psychotherapy to medical students. British Journal of Psychiatry, 148, 670-675.
${ }^{2}$ WILSON, J. (1987) Notes on the dynamics of medical student teaching and implications for future medical practice. Bulletin of the Royal College of Psychiatrists, 11, 194-195.

${ }^{3}$ Berger, N. M. (Ed.) (1978) Video Techniques in Psychiatric Training and Treatment. 2nd edition. New York: Brunner/Mazel.

${ }^{4}$ MAGUIRE, G. P. Teaching psychotherapeutic interviewing to trainees in peripheral hospitals. Talk given at the Spring Quarterly Meeting of the Royal College of Psychiatrists, Manchester, 15-16 April 1987.

${ }^{5}$ AlgER, I. (1978) Freeze framed video in psychotherapy. In Video Techniques in Psychiatric Training and Treatment (ed. N. M. Berger). 2nd edition. New York: Brunner/Mazel. 